\title{
Diabetogenic Drugs and Hormones, what Every Physician should know and be Aware of?
}

\section{Mina T Kelleni*}

Faculty of Medicine, Pharmacology Department, Minia University, Egypt

Corresponding author: Dr. Mina T Kelleni, Faculty of Medicine, Pharmacology Department, Minia University, Egypt, Tel: (+20) 1200382422; E-mail: drthabetpharm@yahoo.com

Rec date: September 25, 2017; Acc date: October 10, 2017; Pub date: October 16, 2017

Copyright: (c) 2017 Khan NT. This is an open-access article distributed under the terms of the Creative Commons Attribution License, which permits unrestricted use, distribution, and reproduction in any medium, provided the original author and source are credited

\begin{abstract}
Much emphasis has been given to drugs managing diabetes but unfortunately, so little research has been devoted to drugs sharing in development and/or progression of diabetes. In the era of poly pharmacy and as the longevity of human beings is expanding together with managing of concomitant chronic diseases for the same patient, it's very likely to find diabetic patients who are receiving one or more of these drugs and after a while, no one can conclude whether these drugs were the cause or they're just deteriorating diabetes. Every physician, internist and endocrinologist should have a good knowledge about drug induced diabetes mellitus including very popular drugs used for managing hypertension, angina, bacterial infection ... etc. and should also try in diabetic and high risk patients to replace them with safer alternatives whenever possible.
\end{abstract}

Keywords: Secondary diabetes mellitus; Thiazide diuretics; Corticosteroids; Propranolol; Streptozotocin

\section{Introduction}

In clinical practice, all internists and endocrinologists should be aware of the possibility of drug induced diabetes mellitus. Poly pharmacy has been a character that is frequently encountered in modern clinical practice and among the prescribed medications; one or more drugs may induce hyperglycemia that may be easily misdiagnosed for diabetes mellitus resulting from either insulin resistance or pancreatic beta cell failure. The crucial importance of detection of drug induced diabetes comes from the fact that this sort of diabetes is usually reversible when the inducing medication is replace or stopped. Some important examples are listed below [1,2].

Thiazide and thiazide like diuretics e.g. Indapamide are very famous group of drugs which induce hyperglycemia. They're frequently prescribed to hypertensive and heart failure patients and during the course of therapy, hyperglycemia may be encountered and eventually type 2 diabetes may develop. In case of chronic use of these drugs, periodic monitoring of serum glucose level is recommended.

Some the traditional beta blockers used in treatment of multiple cardiovascular disorders e.g. propranolol and atenolol are also associated with hyperglycemia. Third generation beta blockers e.g. carvedilol and nebivolol are not associated with this adverse effects and should replace the traditional ones especially in high risk groups e.g. obese patients.

Corticosteroids especially when taken orally or parenteral e.g. prednisone or dexamethasone respectively are well known group of drugs that may induce type 2 diabetes by increasing gluconeogenesis as a result of decreased hepatic insulin sensitivity and by inhibiting peripheral utilization of glucose. Careful monitoring of serum glucose level is a must during a prolonged course of therapy.
Hormonal disturbances other than cortisol may also lead to diabetes mellitus e.g. hypoadrenalism, hypopituitarism as well as clinical and subclinical hypothyroidism are strongly linked with diabetes mellitus and it was recommended to diagnose thyroid dysfunction in type 2 diabetes patients [3].

Some of second generation (atypical) antipsychotics especially clozapine and olanzapine have also been associated with impaired glucose tolerance, new onset type 2 diabetes as well as exacerbation of existing type 1 and type 2 diabetes due to weight gain, change in body fat distribution, or by a direct effect on insulin-sensitive target tissues. Aripiprazole followed by ziprasidone are considered the least risky atypical antipsychotics to be associated with this adverse effect [4].

Some of the respiratory quinolones group of antibiotics e.g. levofloxacin are also associated with hyperglycemia. However, because of the usual short duration of therapy, they're seldom associated with the development of type 2 diabetes.

Some immunomodulators especially tacrolimus are also known to induce hyperglycemia through pancreatic beta cell toxicity, decreased insulin synthesis, and decreased insulin secretion especially in pediatric transplant patients and Afro-Americans.

Protease inhibitors e.g. atazanavir and indinavir used in management of human immunodeficiency virus infected patients have showed a $5 \%$ cumulative incidence of new onset hyperglycemia. This adverse effect has been proved to be an independent one regardless of any other concomitantly used medications.

L-asparaginase used in induction of remission and improvement of long term survival in patients with acute lymphoblastic leukemia has also been associated with hyperglycemia. It decreases insulin synthesis by depletion of asparagine and decreasing insulin secretion from beta cells. Close monitoring for hyperglycemia during L-asparaginase therapy was recommended to enable prompt recognition and early correction [5]. 
Citation: Kelleni MT (2017) Diabetogenic Drugs and Hormones, what Every Physician should know and be Aware of?. Gen Med (Los Angeles)

Page 2 of 2

Pentamidine, an antimicrobial medication used to treat African trypanosomiasis, leishmaniasis, babesiosis, and to prevent and treat pneumocystis pneumonia in immunocompromised patients was also found to be linked to hyperglycemia [6].

In experimental pharmacology, certain drugs are used to induce diabetes in animals e.g. rats. Srteptozotocin is the most famous drug in this category; it's a broad-spectrum antibiotic that is toxic to the insulin producing $\beta$ cells of pancreatic islets. It is currently used clinically for the treatment of metastatic islet cell carcinoma of the pancreas and it offers experimentally a very cost effective and rapid technique to induce diabetes in most strains of rodents [7].

A wise physician should always keep the possibility of secondary diabetes which is drug induced in his/her mind. In my practice I suspect this category in every patient with mild to moderate elevations of fasting or post prandial blood sugar who has been prescribed one of more of the causing drugs. I don't rush to diabetes medications, I generally replace the offending drug, encourage life style and diet modifications and retest the results. In many cases, I find a remarkable improvement of blood sugar levels as soon as few days after replacing the offending drug.

\section{References}

1. Rehman A, Setter SM, Vue MH (2011) Drug-induced glucose alterations part 2: Drug-induced hyperglycemia. Diab Spect 24: 234-238.

2. McEachern R (2017) Steroid/drug-induced hyperglycemia. Clinical advisor, decision spport in medicine, Pediatric.

3. Wang C (2013) The relationship between type 2 diabetes mellitus and related thyroid diseases. J Diabetes Res 2013: 390534.

4. Haupt DW, Newcomer JW (2001) Hyperglycemia and antipsychotic medications. J Clin Psychiatry 27: 15-26

5. Iyer RS, Rao SR, Pai S, Advani SH, Magrath IT (1993) L-asparaginase related hyperglycemia. Indian J Cancer 30: 72-76.

6. Chebane L, Tavassoli N, Bagheri H, Montastruc JL, Centres Régionaux de Pharmacovigilance Français (2010) Drug-induced hyperglycemia: A study in the French pharmacovigilance database. Therapie 65: 447-458.

7. Deeds MC, Anderson JM, Armstrong AS, Gastineau DA, Hiddinga HJ, et al. (2011) Single dose streptozotocin-induced diabetes: Considerations for study design in islet transplantation models. Lab Anim 45: 131-140. 